\title{
Les partis « antisystèmes » en Bolivie : une remise en cause de l'architecture des rapports sociaux?
}

Los partidos «antisistemas» en Bolivia: ¿una contestación de la arquitectura social?

The «anti-system» parties in Bolivia: a protest of the social architecture?

Cécile Casen

\section{(2) OpenEdition}

Journals

Edición electrónica

URL: http://journals.openedition.org/bifea/4655

DOI: 10.4000/bifea.4655

ISSN: 2076-5827

Editor

Institut Français d'Études Andines

Edición impresa

Fecha de publicación: 1 mayo 2006

Paginación: 207-222

ISSN: 0303-7495

\section{Referencia electrónica}

Cécile Casen, «Les partis « antisystèmes » en Bolivie : une remise en cause de l'architecture des rapports sociaux? », Bulletin de l'Institut français d'études andines [En línea], 35 (2) | 2006, Publicado el 08 mayo 2006, consultado el 02 diciembre 2020. URL : http://journals.openedition.org/bifea/4655 DOI : https://doi.org/10.4000/bifea.4655

\section{(ब) $\Theta \Theta$}

Les contenus du Bulletin de l'Institut français d'études andines sont mis à disposition selon les termes de la licence Creative Commons Attribution - Pas d'Utilisation Commerciale - Pas de Modification 4.0 International. 


\title{
Les partis " antisystèmes » en Bolivie : une remise en cause de l'architecture des rapports sociaux ?*
}

\author{
Cécile Casen**
}

\section{Résumé}

L'article analyse l'émergence du MAS et du MIP dans le système de partis bolivien depuis 2000. Le contexte politique de désaffection vis-à-vis des partis politiques «traditionnels » et de remise en cause du « consensus de Washington» ne permet cependant pas à lui seul de comprendre la constitution de ces partis. Issus des mouvements sociaux, leur prétention politique est l'occasion de s'interroger sur le processus de politisation. En quoi le passage du mouvement social au parti politique éclaire-til une architecture sociale qu'il vient ébranler? Que nous révèle-t-il sur les spécificités de la société bolivienne?

Mots clés : Bolivie, partis politiques, mouvements sociaux, politisation

\section{Los partidos «antisistemas» en Bolivia: ¿una contestación de la arquitectura social?}

\section{Resumen}

El articulo analiza la emergencia del MAS y del MIP en el sistema de partidos boliviano a partir de 2000. El contexto político de desconfianza hacia los partidos políticos «tradicionales» y de cuestionamiento del «consenso de Washington», no permite por sí solo comprender la constitución de esos partidos. Salidos de los movimientos sociales, su pretensión política es la ocasión de interrogarse sobre el proceso de politización ¿Cómo el paso de movimiento social a partido político revela una arquitectura social que viene de sacudir? ¿Qué nos revela acerca de las especificidades de la sociedad boliviana?

Palabras clave: Bolivia, partidos políticos, movimientos sociales, politización

* Cette contribution fait suite à mes recherches entreprises dans le cadre du DEA.

** Doctorante à I'IHEAL, 28, rue Saint-Guillaune, 75007 Paris. E-Mail : cecile_casen@yahoo.fr 


\title{
The «anti-system» parties in Bolivia: a protest of the social architecture?
}

\begin{abstract}
This article analyses the MAS and MIP emergence in the system of bolivian parties since 2000. The political context of disaffection with the «traditional» political parties and of questioning the «consensus of Washington» isn't the only element to understand the formation of theses parties. Proceeding from the social movements, its political claim is the opportunity to question the politicization process. How does the transition from the social movement to a political party show a social architecture in a context of shakiness? What does it reveal us about the specificities of the Bolivian society?
\end{abstract}

Key words: Bolivia, Politics parties, social movements, politization

La crise politique bolivienne depuis les années 2000 a donné lieu à une reconfiguration du rapport de force au sein du paysage politique bolivien. De la « guerre de l'eau » d'avril 2000 à la «guerre du gaz» d'octobre 2003, les mouvements sociaux ont imposé leur agenda et donné lieu à l'émergence de nouveaux partis politiques parfois appelés «antisystèmes ». Les élections présidentielles de 2002 sont marquées par leur montée en puissance. Le Movimiento Indígena Pachakuti ${ }^{1}$ (MIP) et le Movimiento al Socialismo (MAS) sont issus de la structuration de mouvements sociaux et se présentent comme leur « instrument politique ».

Notre interrogation porte sur la nature de ces partis du fait de leur origine sociale. L'approche en terme d'architecture sociale nous est apparue intéressante pour problématiser le passage du mouvement social au parti politique. Jacques Lagroye parle en effet de l'existence d'espaces a priori différenciés, qu'ils soient politiques, sociaux, culturels, religieux ou privés. Il les désigne comme «ordres d'activités spécialisés (...) historiquement différenciés, institutionnalisés, leur distinction et leur séparation acquérant un statut de naturalité dans l'évidence des règles et des rôles qui caractérisent chacun d'entre eux. »(Lagroye, 2003 : 362). L'agencement de ces différents ordres renvoie, entre autres, à la distinction entre organisations sociales (syndicats, coordinations) et institutions politiques. Si l'État tente de maintenir la fiction d'une frontière stricte entre revendications sur les conditions de vie et revendications proprement politiques, en quoi le positionnement bivalent des acteurs et le «mélange des genres » qu'ils pratiquent remettent-ils en cause cette « architecture des rapports sociaux» (Lagroye, 2003 : 363) ? En quoi la politisation des mouvements sociaux vient-elle contredire le mode d'articulation entre l'État et la société mis en place depuis la transition à la démocratie et, dans le même temps, concourt à la formation de partis politiques d'un genre nouveau?

Afin de le comprendre, nous commencerons par resituer le contexte qui préside à la montée en puissance de mouvements contestataires. Comment le MAS et le MIP parviennent-ils à s'insérer dans I'appareil institutionnel ? En quoi questionnent-ils le fonctionnement de la « démocratie pactée »?

1 Pachakuti se réfère en quechua/aymara à une transformation liée à un renversement du temps et de l'espace. La notion la plus proche dans le paradigme occidental est celle de révolution. 


\section{CONTEXTE POLITIQUE ET INSTITUTIONNEL}

Depuis vingt ans les nations latino-américaines ont tourné la page des gouvernements militaires pour accéder à la démocratie. Cependant, Georges Couffignal relève qu'il s'est agi plus précisément d'une «double transition» : à la démocratie comme régime et à l'économie de marché. La confusion entre les dimensions politique et économique s'impose d'autant plus facilement que les gauches latino-américaines, parce qu'elles sont en partie privées de la référence au modèle social-démocrate, pâtissent douloureusement de l'échec du socialisme soviétique. À la « décennie perdue $2^{2}$ succèdent timidement, dans les années 1990, les premières remises en cause de l'exercice d'une démocratie qui ne profite pas assez à ses citoyens. En effet, le passage du modèle de développement économique fondé sur la substitution des importations à un modèle exclusivement orienté vers l'exportation représente un coût social considérable. À cet égard, le même auteur signale aussi l'absence de contrôle démocratique puisque «ni les parlements (faibles), ni les partis (en crise ou en complète recomposition), ni les syndicats (déconsidérés ou très affaiblis), ni les organisations de la société civile (peu présentes dans le champ politique), n'étaient en mesure de contraindre [ses] élites à gouverner autrement 》(Couffignal, 2003 : 229). Si la majorité des latino-américains soutiennent la démocratie en tant que régime, ils se montrent néanmoins de plus en plus critiques à l'égard de ses effets sociaux.

La Bolivie est le premier pays latino-américain revenu à la démocratie à appliquer un plan d'ajustement structurel. L'échec du gouvernement de la UDP (1982-1985) «arrastró consigo a toda la izquierza política » (Calla, 1993 : 71). Le pays s'engage dès 1985 dans l'expérience néolibérale. Le démantèlement de l'État corporatiste est d'autant plus violent en Bolivie que le capitalisme étatique y figurait parmi les plus développés du continent. Laurence Whitehead rappelle ainsi qu'« en dehors de Cuba, la Bolivie des années 60 et 70 était probablement le pays latino-américain dans lequel la participation de l'État à l'économie était la plus considérable» (Crabtree \& Whitehead, 2000).

Parallèlement, la refondation du système démocratique permet d'assurer pour la première fois en Bolivie, une certaine stabilité politique et institutionnelle. Des réformes politiques améliorent progressivement les mécanismes de représentation et un système de partis — c'est-à-dire un ensemble relativement stable de partis politiques qui interagissent selon des règles acceptées par tous - se met en place. La démocratie devient the only game in town ${ }^{3}$. Les partis politiques boliviens détiennent désormais le monopole de la représentation. D'une part, les militaires sont rentrés dans leurs casernes. D'autre part, la crise traversée par la COB (Central Obrera Boliviana) et le mouvement ouvrier prive le syndicat de son rôle historique. Depuis 1989 et pendant plus de dix ans, le système de parti fonctionne de façon stable, orchestré par cinq organisations : le MNR, I'ADN, le MIR, I'UCS, et la Condepa. Les trois premiers partis voient leurs leaders accéder à la Présidence de la République et, même si les deux derniers prétendent représenter une opposition, ils n'hésitent pas à participer à des coalitions gouvernementales. II existe donc un foyer idéologique capable de structurer les pratiques et les discours des acteurs politiques.

La succession des gouvernements pendant 20 ans, suivant des processus électoraux transparents, constitue un démenti à l'instabilité chronique qui semblait devoir toujours caractériser ce pays (Lavaud, 1991).

Pourtant, le régime démocratique ne parvient pas à faire face à la croissance des inégalités et de la pauvreté. Le discours officiel et public sur la démocratie exacerbe la perception de l'écart entre idéal affiché et réalité du système. Censées offrir un large accès à la représentation, les

2 On désigne ainsi les années 1980 en Amérique latine par référence à l'augmentation de la pauvreté qui sévit sur l'ensemble du continent.

3 Pour Juan Linz (1990) la consolidation démocratique est achevée sous réserve de remplir deux conditions. Premièrement, les acteurs politiques majeurs (partis, syndicats ou toute autre institution) ne doivent pas envisager l'existence d'une alternative aux processus démocratiques pour obtenir le pouvoir. Deuxièmement, ils n'opposent pas leur veto à l'action des preneurs de décision démocratiquement élus. 
institutions démocratiques ne tiennent pas leurs promesses et sont de plus en plus vivement critiquées. Le recours routinier aux pactes entre partis répond à la nécessité de stabiliser les institutions de la jeune démocratie mais contribue finalement à la vider de son sens. Dans le même temps, le passage sur la scène internationale d'une approbation unanime du « consensus de Washington $»^{4}$ à ce qu'on a pu appeler la Washington confusion encourage la contestation. La crise politique bolivienne des années 2000 apparaît comme une illustration significative de cette remise en cause. Les vagues de mobilisations constituent une réaction de défi lancé à une démocratie dont l'unique vertu serait d'avoir doté le pays d'une stabilité politique propice à la mise en place d'un modèle économique hégémonique. Dans une enquête réalisée en 1993 (Soria Soravia, $2002: 86$ ), seulement $16 \%$ des personnes interrogées considèrent que les partis politiques représentent l'ensemble du pays, contre $77 \%$ qui estiment qu'ils ne représentent que certains groupes. Cette absence de légitimité s'accompagne d'un fort soutien populaire à la démocratie comme régime. Paradoxalement, la majorité des citoyens croient que les partis sont essentiels en démocratie tout en leur refusant leur confiance.

Si donc le régime politique propose un modèle de légitimité efficient qui assure une relative stabilité institutionnelle pendant plus de vingt ans, la vague des mobilisations populaires d'avril 2000 à octobre 2003 le remet néanmoins fortement en cause. Avec Sydney Tarrow, on peut y voir un cycle de mobilisation, c'est-à-dire « une phase d'intensification des conflits et de confrontation dans le système social, qui inclut une rapide diffusion de l'action collective des secteurs les plus mobilisés aux moins mobilisés. » (Tarrow, 1997 : 142). Vingt ans après la transition vers la démocratie, alors que les institutions démocratiques sont a priori consolidées et fonctionnent de façon routinisée, les mouvements sociaux interviennent avec force et sont à l'origine de la crise politique. Tout d'abord, en 2000, la guerre de l'eau de Cochabamba voit la victoire du mouvement social contre l'entreprise américaine Bechtel chargée par le gouvernement de la privatisation de cette ressource. Parallèlement, la répression et la militarisation croissantes dans la région du Chapare ont conduit à parler là encore de « guerre »5 de la coca. Celle-ci est à l'origine de la montée en puissance du mouvement cocalero mené par le jeune dirigeant Evo Morales. S'ajoutent à ces deux foyers de tension les bloqueos aymaras de l'altiplano qui commencent par un cerco (encerclement, siège) de La Paz en avril 2000 et se poursuivent par intermittence tout au long de l'année 2001. En octobre 2003 « la guerre du gaz » semble mettre fin à un cycle de mobilisations en entraînant la chute du président en exercice, Sanchez de Lozada. La mise en place d'un nouveau gouvernement autour de son Vice-Président Carlos Mesa « conclut le cycle de la démocratie pactée $» 6$.

La guerre de l'eau de Cochabamba semble ainsi agir comme une expérience fondatrice pour les mouvements contestataires qui lui font suite. La victoire de David sur Goliath7 donne à voir la vulnérabilité du modèle néolibéral qui se veut tout puissant et ouvre la brèche de sa remise en cause. Par opposition à l'hypothèse de la prétendue faiblesse politique des pauvres qui seraient condamnés à « souffrir en silence ${ }^{8}$, la victoire de Cochabamba fonctionne comme un symbole de l'efficacité des mouvements sociaux et donne lieu à la structuration d'une communauté

4 Terme créé en 1989 par l'économiste John Williamson : «I made a list of ten policies that I thought more or less everyone in Washington would agree were needed more or less everywhere in Latin America, and labeled this the "Washington Consensus"». Cf. A Short History of the Washington Consensus, disponible en ligne. Le consensus porte entre autres sur l'ouverture des frontières et la réduction du rôle de l'État dans l'économie, la privatisation des entreprises publiques, I'abandon des subventions aux produits ou services, la suppression des déficits budgétaires.

5 Elle s'inscrit par ailleurs dans la « guerre contre les drogues » engagée au niveau international. Selon le rapport de la Defensora del Pueblo, Ana María Romero, le conflit cocalero est à l'origine entre 1987 et 2002 de 57 cocaleros morts, 500 blessés par balle et 4000 détenus en attente de procès.

6 «Carlos Mesa gobierna sin mayoria parlamentaria que pueda ser utilizada como rodillo, y con ministros apartidistas » (Alenda, 2004).

7 Selon les termes de Assies (2001).

8 Pour une discussion sur ces hypothèses, cf. O'Donnell (1996). 
d'analyse, d'un diagnostic partagé sur qué hacer. Dès lors, ces mouvements créent des conditions propices à la formation d'un esprit et d'une conscience populaire critiques, d'un personnel militant, de structures et d'organisations capables de coordonner efficacement des actions de nature politique. Les journées d'octobre 2003 connues comme « la guerre du gaz » sont souvent présentées comme la mobilisation la plus massive depuis la Révolution Nationale.

Or, le MIP et le MAS sont issus de ces mouvements sociaux et des organisations qui les structurent. En retour, les résultats électoraux obtenus par ces deux organisations partisanes « antisystèmes » lors des élections présidentielles de 2002 traduisent l'ampleur des mobilisations. Le MAS arrive en effet à moins de deux points de différence, le MNR devenant ainsi la deuxième force politique du pays. Pour beaucoup, le score du MIP est également une surprise. Alors que les partis indianistes qui l'ont précédé n'ont jamais dépassé les $3 \%$ de votes au niveau national, le MIP parvient à en réunir plus de $6 \%$. Leur insertion dans le système de partis entraîne sa reconfiguration et son bouleversement. Elle implique une polarisation du champ politique par opposition à la situation antérieure où la logique centripète prévalait. Dans la mesure où les deux partis incarnent la critique des pratiques de la démocratie « pactée » et où ils refusent de prendre part à son jeu, ils assument la qualité $\mathrm{d}^{\prime}$ « antisystème ». Après vingt ans de consensus programmatique, de pacification de la vie sociale et de «sommeil » des forces de gauche, le champ politique se charge à nouveau d'une conflictualité que l'on s'efforçait de refouler.

\section{UNE NOUVELLE ARTICULATION ENTRE L'ÉTAT ET LA SOCIÉTÉ ?}

Pour Jean Blondel, au fondement de tout parti politique, on décèle la présence d'un conflit social profond. L'histoire montre comment, en Europe, les partis socialistes sont nés de la poussée des mouvements ouvriers et syndicaux. Pourtant, malgré la richesse des interactions entre les facteurs politique et social de la vie publique, peu de travaux universitaires se sont attachés à la question de leurs intrications. Nombreux en revanche sont ceux qui, traitant de l'un ou de l'autre de ces facteurs, évoquent ponctuellement leurs interactions. Une explication de cette absence d'analyse globale est à chercher, en partie, dans le cloisonnement artificiel des disciplines. Les partis constituent, en effet, un objet canonique de la science politique tandis que les mouvements sociaux relèvent traditionnellement de la sociologie. En introduction à son ouvrage paru en 2003 sur les interactions entre mouvements sociaux et partis politiques, Jack Golstone n'hésite pas à écrire en réaction à cette pratique universitaire que « les partis politiques et les mouvements sociaux ne peuvent être appréciés sans leur intime relation.» (Golstone, 2003 : 2). L'origine militante des dirigeants du MIP et du MAS nous invite ainsi à poursuivre le projet de recherche proposé par Michel Offerlé à partir de « la nécessité de désenclaver l'objet parti politique. » (Offerlé, 2002 : 4). En effet, si le parti est agent du conflit en tant que tel, il est aussi l'instrument de son intégration politique.

Par ailleurs, I'hypothèse d'un lien organique entre les mouvements sociaux et les formations partisanes qui nous occupent implique d'envisager le politique du point de vue de la question de la légitimité par opposition à la réduction de la démocratie à la seule procédure électorale. Contre I'assimilation des mouvements sociauxà un désordre relevant d'une « pathologie sociale. » (Neveu, 2002 : 3), ils constituent un ingrédient nécessaire à la légitimation d'un pouvoir démocratique. Du point de vue du chercheur pourtant, un «travers réside dans une fascination pour l'objet, une impatience à théoriser l'immédiat, qui débouche parfois sur une célébration complice de la nouveauté. » (Neveu, 2002 : 71). Comprendre le rôle des mouvements sociaux implique aussi de se défaire de cette vision qui oppose «l'univers de l'organisé, régi par des règles et des procédures fermes, à l'effervescence créatrice et confuse des mouvements sociaux. » (Neveu, 2002 : 71). En effet, la dimension institutionnelle n'est pas absente de leurs modalités d'action. Se pose au contraire de façon cruciale la question de leur organisation. L'existence de nos partis en est la meilleure preuve. Par conséquent, il ne s'agit ni de disqualifier ces mouvements ni de s'en faire les admirateurs béats mais de tenter de comprendre dans quelles mesures ils nous 
permettent d'analyser la nature des partis politiques qui en sont issus. Nous retenons la définition de François Chazel selon laquelle un mouvement social est « une entreprise collective de protestation et de contestation visant à imposer des changements — d'une importance variable — dans la structure sociale et/ou politique par le recours fréquent — mais pas nécessairement exclusif — à des moyens non institutionnalisés. » (Chazel, 1993 : 270).

Le terme $d^{\prime}$ " antisystème » quant à lui, se révèle polysémique et dans une certaine mesure contradictoire. D'une part, il est assumé par le MIP et le MAS qui y voit le principe de leur démarcation à l'égard des autres formations partisanes. Ils prétendent incarner la critique des pratiques de la démocratie « pactée »9 et refuser de prendre part à son jeu, et pourtant, leur constitution en parti politique indique bien une volonté de participer des institutions démocratiques. D'autre part, ce qualificatif résulte d'une appréciation émise par leurs détracteurs qui assimilent leurs leaders respectifs I'un à un terroriste (Quispe), l'autre à un narcotrafiquant (Morales) (Guzman \& Orduna, 2002). Georges Lavau a analysé les effets favorables que la contestation d'un système donné peut apporter à la survie du même système. La «fonction tribunitienne» remplie selon lui par le Parti communiste français correspond à celle dévolue dans la République romaine au tribun de la Plèbe, à savoir exprimer le mécontentement et la grogne des exclus. Elle permet d'assurer la survie du système en déviant «les virtualités révolutionnaires ». On peut ainsi se demander si contrairement à ce que laisse sous-entendre la catégorie de "partis antisystèmes » l'existence de ces partis n'est pas un signe de la bonne santé de la démocratie bolivienne, de ses capacités de représentation, médiations et expressions des intérêts de la société. Si l'activité partisane est la forme de participation «systémique», alors qualifier un parti d'« antisystème » apparaît comme contradictoire. Se constituer en parti suppose a priori l'acceptation des institutions et non leur subversion. Le passage du mouvement social au parti politique implique une assimilation des règles du jeu démocratique par ceux qui aspirent à y participer plus qu'une volonté d'y mettre un terme.

Le MIP et le MAS se nourrissent et cristallisent à la fois la contestation. Mais assumer un rôle parlementaire en tant $q^{\prime}$ '«instrument politique» de mouvements extra-parlementaires implique de toujours se situer dans l'entre-deux d'espaces différenciés. Alors que les élections de juin 2002 n'ont pas encore œuvré à sa légitimation politique, une déclaration d'Evo Morales apparaît significative des tensions qui s'exercent entre des engagements multiples et parfois contradictoires :

«Para mí el tema del voto pasa a un segundo plano, creo más en las luchas sociales, porque con las marchas y bloqueos cambiamos leyes, anulamos decretos, hacemos aprobar leyes (...) el Parlamento sirve para ser expulsado y donde la gente puede tener idea de qué son los partidos tradicionales (...) nosotros vamos con otra mentalidad, contra el modelo y el sistema, tal vez estando dentro del sistema (...) combinando la lucha parlamentaria con la lucha social. » (La Prensa, 2002).

Selon René-Antonio Mayorga, «Con el MAS y el MIP se ha replanteado temas de la izquierza tradicional como el anti-imperialismo, la defensa de los recursos naturales, la recuperación de la economía estatal etc. » (Mayorga, 2002). Cependant, le MIP et le MAS proposent deux critiques différentes de la démocratie pactée et incarnent en ce sens deux manières d'être " antisystème ». Ils assument ainsi un positionnement très différent dans leur relation avec les institutions démocratiques.

Le MIP est un parti indianiste. Depuis les années 1970, ce courant de pensée s'oppose à I'indigénisme. S'ils portent tous les deux sur la question de l'Indien, le premier est énoncé par un locuteur qui revendique son indianité et dénonce le second comme un «indigénisme sans indien », un « indigénisme de salon, de blancs pour les blancs »10. Felipe Quispe fonde son action

9 Principe de gouvernement qui repose sur la préséance des « pactes » et la formation de coalitions gouvernementales. Il caractérise le fonctionnement démocratique depuis 1985.

10 Ramiro Reinaga, fils de Fausto Reinaga (In : Barnadas, 1983). 
politique sur l'identification ethnique, et s'inscrit dans la tradition discursive de l'indianisme radical, comme en témoigne son insistance à distinguer l'indien du q'ara11. Dénommé le «Mallku»12, il se présente comme le leader de la nation aymara et aspire à un autogobierno indio, ce qui le conduit à rejeter les institutions libérales de la nation bolivienne. Ce rejet se traduit dans les années 1990 par son engagement de guérillero au sein de Ejército Guerillero Tupac Katari. Pourtant, et c'est bien là un des aspects de la tension qui existe entre principes et pratique, il est aujourd'hui Secrétaire Exécutif du principal syndicat paysan bolivien (CSUTCB) et siège au Parlement depuis 2002. Cette tension est d'autant plus patente que cette vie « officielle » ne contrevient pas à la poursuite d'actions directes visant à faire pression sur l'État.

De son côté, le MAS est un parti composite dont la ligne idéologique reste floue. À la différence du MIP, le MAS ne porte pas un discours en faveur d'un gouvernement indigène autonome mais ne renonce pas, loin s'en faut, à recourir à cette dimension. II s'efforce de proposer une synthèse entre l'idée du Pachakuti andin et le marxisme, entre la tradition guevariste et la mouvance altermondialiste. Né au Chapare des mobilisations contre la loi 1008 (depuis 1988 elle planifie l'éradication des cultures de coca), le MAS est d'abord conçu comme une extension des instances syndicales paysannes, à savoir comme leur « instrument politique ». Pourtant, le succès électoral national de juin 2002 s'explique par sa capacité à réunir des suffrages au-delà des paysans cocaleros. Fruit du vide laissé par la méfiance populaire envers la classe politique «traditionnelle», ses résultats significatifs indiquent aussi la nouvelle articulation d'un discours suffisamment vague pour rassembler largement au-delà des fractures de la société bolivienne.

\section{MÉTAMORPHOSES DU SYNDICAT : LA FIN DU « CENTRALISME OUVRIER » ET LE REPOSITIONNEMENT FACE À L'ÉTAT}

Les acteurs mobilisés depuis 2000 sont les cocaleros du Chapare et des Yungas et les comunarios aymaras de I'Altiplano. Leur caractère essentiellement rural est significatif de la métamorphose des luttes sociales. L'histoire des relations tumultueuses entre les mouvements ouvrier et paysan depuis la révolution de 1952 excède largement le cadre de cet article et il nous est impossible de la développer ici. Qu'il nous suffise cependant de rappeler que jusque dans les années 1970, la Central Obrera Boliviana, en tant que syndicat ouvrier, a exercé son hégémonie sur le syndicalisme paysan en grande partie coopté par l'État national-populaire. "La 'centralidad obrera' para las orientaciones y accionares de los movimientos populares en el país » (Calla, 2000 : 216) a tendu à marginaliser le mouvement paysan au sein de l'instance syndicale. Pourtant, le changement de tendance s'amorce, d'abord dans la clandestinité, ensuite au grand jour avec la montée en puissance du katarisme. Ce courant s'impose en effet comme une référence au sein du syndicat paysan et lui redonne de sa combativité. L'altiplano aymara qui a développé une résistance plus fondamentale au projet d'intégration nationale est le foyer le plus actif du nouveau discours indianiste. En 1971, la fondation de la CSUTCB formalise l'inscription du katarisme dans la formation d'un syndicat paysan autonome. La nouvelle instance syndicale affirme son indépendance à l'égard de l'État et sa vocation à représenter l'ensemble du monde rural bolivien. Cependant, ce sont des kataristes aymaras qui sont à l'origine du syndicat et qui le dirigent. Ainsi, s'illustre son « andinocentrismo » (Calla, $1993: 63$ ).

À partir de 1985, un nouveau coup est porté à la prédominance du mouvement ouvrier avec la privatisation de l'exploitation minière et le licenciement massif des mineurs qu'elle entraîne. Au-

11 En quechua et en aymara, q'ara désigne de façon péjorative les blancs et les métisses. Il signifie à la fois « pelé » et «nu » et renvoie à l'absence de culture andine et d'éthique qui les conduit à voler et à exploiter. Cf. Albo, $2002: 80$.

12 Signifie condor en aymara/quechua et désigne normalement l'autorité traditionnelle au niveau des instances communautaires ou ayllus. 
delà de sa portée économique, cette mesure est lourde de conséquences sur le plan politique puisqu'elle affaiblit fatalement la FSTMB13. Alors que cette dernière constituait le secteur leader au sein de la $C O B$, privée dans un premier temps d'une grande partie de ses membres, elle amorce très largement son déclin. En août 1986, le mouvement ouvrier trouve néanmoins les ressources d'organiser la Marcha por la Vida y por la Paz visant en réalité la défense des mines «publiques». Elle représente la dernière grande mobilisation ouvrière en Bolivie. À partir de cette date, la COB voit donc sa capacité de mobilisation et de proposition - qui caractérisait jusqu'alors son « autocentrismo» (Calderón \& Laserna, 1989) — s'effondrer et avec elle le modèle syndical d'articulation de l'État à la société. Après un temps de latence, l'ancienne hégémonie ouvrière se déplace. Elle migre du mouvement ouvrier vers le mouvement paysan-indigène, alors même que la structure syndicale est délégitimée en tant qu'acteur référent face à l'État.

Jean-Pierre Lavaud voit dans la période antérieure au gouvernement de I'UDP (1982-1985) un moment qui «se asemeja a la época del MNR, de la cual es, realmente, continuación y fin » en ce sens que «se halla marcada por el juego competitivo de la clase política y de la COB». La formule du cogobierno COB/MNR qui caractérisait les premières années de la Révolution est un mécanisme de représentation original. En effet, dans ce cadre, les dirigeants syndicaux sont nommés membres du gouvernement aux côtés des membres du parti. Les années 1985 marquent la fin de cette conception de l'État, dans la mesure où désormais, «La escena política es ocupada por la alianza de la clase política y de una neo-oligarquía reconstituida que corona el sector privado de la economía. » (Lavaud, 1993 : 50). On pourrait ajouter avec Fernando Calderón que :

«Sous le gouvernement UDP prit fin la culture politique de l'affrontement héroïque [...] entre les forces armées et la FSTMB et les grands mouvements historiques. Ces derniers ne purent reconduire dans les espaces de pouvoir ce lien si créatif, si particulièrement bolivien, entre les formes d'action et d'auto représentation socioculturelle directe, et la démocratie institutionnelle représentative. » (Calderón, 2001 : 438)

La vision de la société bolivienne en terme de conflit de classe laisse place à la célébration d'un consensus démocratique reconnaissant la diversité culturelle. L'État, jusqu'alors partie prenante du conflit de classe, se transforme en arbitre entre les intérêts divergents d'une société pacifiée. II se montre toujours plus enclin à déléguer au citoyen et à la société civile une plus grande partie de ce qui jusqu'alors semblait devoir relever de son domaine de compétence. L'utilisation d'une rhétorique conciliatrice, bien qu'elle soit conçue comme la pierre de touche d'une nouvelle ère démocratique exempte de conflits, ne résiste pas à l'épreuve des faits. L'entrée en scène des mouvements sociaux oppose en effet un démenti à la pacification de la vie sociale en ce qu'elle replace la question hégémonique au centre de ses luttes, induisant ainsi à nouveau une logique de conflictualité. En quoi les revendications des mouvements sociaux débordent-elles en un sens politique les modes d'insertion dans la société globale prévus par l'État?

\section{LA RHÉTORIQUE DE LA «PARTICIPATION »}

La fin du modèle de l'État national-populaire implique une véritable réforme de la citoyenneté. Le projet de modernisation de l'État se traduit ainsi dans les années 1990 par l'adoption d'un ensemble de réformes. Nous citons les principales. La réforme constitutionnelle de 1994 consacre la reconnaissance du caractère multiethnique de la nation bolivienne. La même année, la Loi de Participation Populaire (LPP) engage un processus de décentralisation par municipalisation. La réforme électorale établit les districts uninominaux.

À travers la reconnaissance par la Constitution des Tierras Comunitarias de Origen (TCO) et I'instauration par la LPP des Organizaciones Territoriales de Base (OTB), ces réformes encouragent

13 Federación Sindical de Trabajadores Mineros de Bolivia. 
Les partis « antisystèmes » en Bolivie : une remise en cause de l'architecture des rapports sociaux

la création d'espaces politiques locaux. La LPP modifie notoirement le scénario politique en élevant la municipalité à une catégorie de premier ordre14. La nouvelle distribution des sièges favorise les partis faibles au niveau national mais qui jouissent néanmoins d'une crédibilité locale ou régionale. Cette évolution significative de l'environnement institutionnel influe logiquement sur la structuration du MIP et du MAS qui lui sont en partie redevables.

Pourtant les mouvements sociaux tout en s'appropriant les outils offerts par cette « démocratisation par le haut» se mobilisent contre le caractère formel de cette "participation ». Comment expliquer ce paradoxe?

La LPP et la réforme multiculturelle ont été présentées par les acteurs étatiques comme une réponse et une prise en compte des exigences indigènes. Pourtant, elles ne sont pas le fruit d'une négociation avec un mouvement social encore balbutiant au début des années 1990. Elles apparaissent plutôt comme des mesures relevant de l'application du modèle démocratique promu par les grands organismes internationaux (FMI-Banque Mondiale). L'émergence des mouvements sociaux sur la scène politique s'inscrit dans le nouveau paradigme des réformes tout en le contestant. Pour cette raison, la crise politique doit être analysée à partir des caractéristiques du nouveau modèle d'articulation entre État et «société civile». Il se caractérise d'abord par l'ébranlement définitif du modèle syndical de médiation politique, ensuite par la mise en place d'un nouveau mode de relation entre l'État et la « société civile».

«L'objectif de la participation populaire est de donner la parole à chaque membre des OTB, et que celles-ci occupent l'espace qui jusqu'à présent a été celui des comités civiques, des syndicats, de la $\mathrm{COB}$ et de tout autre petit groupe se proclamant représentant de la majorité. Ces organisations devront s'adapter à la loi pour survivre. $\gg^{15}$.

Ainsi, la légitimité d'interlocuteurs des centrales syndicales et en particulier de la CSUTCB est battue en brèche.

«Dans le cadre de notre proposition, la CSUTCB ne pourrait pas participer en tant qu'organisation indigène. La spécificité des Ayllus repose sur la tradition, il s'agit de communautés qui maintiennent des systèmes d'élections traditionnelles. La CSUTCB est un syndicat, elle a un système complètement différent de désignation des dirigeants. $\gg 16$.

La LPP répond en fait aux revendications exprimées lors de la marche «por el territorio y la dignidad» (1990) des indigènes des basses terres. Longtemps marginaux au sein du champ politique bolivien, cette marche marque leur irruption sur la scène nationale et leur volonté d'être prise en compte par l'État. La LPP semble ainsi s'adresser plus à eux qu'aux populations d'origine amérindienne des Andes considérées avant tout comme « paysannes ». Dans un entretien réalisé par la même chercheuse avec le vice-ministre des Affaires indigènes, à la question de savoir si les populations syndiquées des Hautes Terres seraient des indigènes, il répond :

- Non, ce sont des paysans.

- Pourtant, ils affirment leur identité indigène...

- Ils sont aymaras et quechuas, bien sûr, mais leurs besoins, leurs préoccupations, ne sont pas celles de peuples indigènes. Dans le cadre de ce vice-ministère, j'ai donc très peu à faire avec la CSUTCB17.

\footnotetext{
14 Georges Couffignal insiste ainsi sur le caractère audacieux des lois boliviennes de décentralisation « en matière de transferts de compétences et de transferts financiers au bénéfice des communes.» (Couffignal, $2003: 231$ )

15 Consultant de la Secretaría de Participación Popular, La Paz, 1996 (In : Lagos, 2001 : 274).

16 Entretien avec Rodrigo Jemio, Technicien de la direction Coordination avec la coopération internationale du MAIPO, 29 juillet 2004 (Raous, 2004).

17 Entretien avec Enrique Camargo Manuel, vice-ministre du MAIPO, le $1^{\text {er }}$ avril 2002 (Raous, 2004).
} 
Dans la perspective officielle, la Centrale syndicale devient ainsi « une forme d'organisation de plus, que le contexte oblige à entrer en compétition avec d'autres pour la représentation des intérêts du «peuple» face à l'État. » (Calla, 2000 : 216). Ricardo Calla affirme que :

« par opposition à la période précédant l'ajustement, pendant laquelle la COB tendait à concentrer et à condenser l'action des réseaux syndicaux en Bolivie [...], aujourd'hui le syndicalisme bolivien apparaît comme un réseau déconcentré, d'orientation régionale, différenciée et diversifiée sur le plan sectoriel, à tel point qu'il est nécessaire de parler de syndicalismes et non plus d'un syndicalisme bolivien. »(Calla, $2000: 217$ ).

Face à cette perte de pouvoir, on comprend que la CSUTCB dénonce la LPP comme une manœuvre destinée à miner sa propre représentativité et sa capacité de médiation. Elle la qualifie alors de «ley maldita»

On retrouve cette fragmentation de l'espace politique dans la manière dont les gouvernements successifs conçoivent la négociation avec les acteurs mobilisés. Les institutions démocratiques sont conçues comme le cadre devant permettre l'épanouissement du dialogue entre l'État et la «société civile». Maria L. Lagos souligne ainsi que «la LPP est envisagée comme le moyen de canaliser ce qui est défini comme des formes politiques de confrontation, au travers d'expressions nouvelles, légitimes et consensuelles» (Lagos, 2001 : 274). Lors du conflit à Cochabamba, la Coordinadora del agua s'impose comme l'interlocuteur central pour le règlement du conflit alors que son existence institutionnelle est quasi nulle ${ }^{18}$. Le comité civique de Cochabamba jouit au contraire d'une légitimité institutionnelle mais il est ouvertement dénigré par la population. «Surgie de nulle part», selon les mots de Laurence Whitehead (Crabtree \& Whitehead, 2000), la Coordinadora apparaît à la faveur de l'absence d'interlocuteurs légitimes aux yeux de la population pour négocier avec le pouvoir. Ce divorce entre les institutions et les citoyens éclaire aussi l'incompréhension mutuelle concernant la conception des modalités de « participation ». Cette dernière est en effet cantonnée à des demandes sectorielles.

Cette restriction est observable à plusieurs reprises au cours de la crise des années 2000. En septembre 2000 par exemple, alors que le pliego petitorio émis lors des bloqueos comprend entre autres l'annulation du décret 2106019, la CSUTCB obtient à la suite des négociations la promesse de la distribution de mille tracteurs. La création du ministère des Affaires indigènes et des Peuples originaires (MAIPO) est alors critiquée par Víctor-Hugo Cárdenas « como una vuelta a la visión reduccionista y solo sectorial de la problemática campesino indígena, en vez de considerarla com un ingrediente central de todo el quehacer público.» (Albo, 2002 : 85). Par ailleurs, lors des mobilisations de juillet 2001, organisées par la CSUTCB, le Gouvernement refuse de négocier sur la base des revendications présentées par les dirigeants car elles concernent notamment un changement des symboles républicains (drapeau, héros fondateurs) pour les figures de Bartolina Sisa, Tupak Katari et la Whiphala. Dans la mesure où ces requêtes ne concernaient pas la réalité agraire

«Le Ministre de la Présidence, Pérez Monasterios, a affirmé que le Gouvernement n'engagerait pas de négociation sur la base de telles demandes, les qualifiant de proclamation politique qui ne concerne pas le développement rural du pays. » (El Deber, 2001).

Ces épisodes permettent d'illustrer le type de sollicitations susceptibles d'être prises en compte, à savoir celles concernant directement les intérêts corporatifs et sectoriels des paysans de I'altiplano, dans le cadre d'une négociation entre l'État et un secteur particulier. L'État réduit les demandes des «Indigènes » à ce qui relève de l'espace de leur communauté. Quand elles débordent ce cadre et intéressent des choix de société plus globaux, les portes se ferment.

18 Le décret qui instaure l'état de siège stipule que "groups without any legal basis whatsoever are claiming to represent the people» violant ainsi la Constitution. In : Assies (2001).

19 Décret de 1985 qui consacre la libéralisation de l'économie et la privatisation des entreprises d'État. Il correspond au plan d'ajustement structurel. 
Les partis « antisystèmes » en Bolivie : une remise en cause de l'architecture des rapports sociaux

«Les organisations ont été reconnues, ainsi que leurs manières propres de concevoir la participation. Mais elles ne sont pas envisagées comme aptes à avoir des idées propres sur l'État. Leurs positions ne peuvent concerner que leur propre secteur. Dans ce contexte, le fait que les mouvements sociaux aient abandonné leurs demandes sectorielles gêne tout le monde. $\gg 20$.

Le mouvement social outrepasse les modalités d'insertion dans la société globale prévues par les réformes des années 1990. D'une façon générale, les représentants de l'État tendent à attribuer les impasses successives sur lesquelles débouchent les mobilisations sociales, non pas tant à leur caractère sectoriel, qu'à leur radicalité21. De plus, si l'on reprend la définition proposée par Oscar Olivera, selon laquelle la Coordinadora est un « frente amplio que concentra sectores sociales $\gg 22$, il apparaît clairement qu'elle a vocation à représenter au-delà des limites d'un seul secteur. De même, en janvier 2003, les mobilisations cocaleras donnent lieu à la formation d'une instance de coordination entre les mouvements sociaux (el Estado Mayor del Pueblo). Gonzalo Sánchez de Lozada tente de s'y opposer :

«Le Président s'est adressé hier aux cocaleros et aux autres secteurs sociaux pour leur demander de ne pas organiser une " association des créanciers ", en réponse à l'intention qu'Evo Morales a affiché de former un seul bloc de pression sociale. Il a affirmé qu'il ne négocierait qu'avec chacun des secteurs de façon séparée, et n'accepterait pas le dialogue en bloc, qui selon lui relève d'une position clairement politique. » (La Razón, 2003)

Ainsi, lorsque les acteurs sociaux se mobilisent au nom de revendications qui sortent de leur assignation sectorielle, leurs actes sont dénoncés comme illégitimes car «politiques. » Cette assignation pervertit donc le discours de «la participation» en le confinant dans les étroites limites d'un seul « ordre d'activité » celui du syndicat corporatiste ou sectoriel.

Si la LPP permettait réellement ce qu'elle prétend reconnaître, à savoir la participation, la crise politique ne revêtirait probablement pas la forme aiguë qu'elle prend actuellement. Or, la participation apparaît plus formelle que réelle à ceux qu'elle concerne. Cette réalité est sensible dans les témoignages de la rue puisqu'on peut y entendre que "lorsque nous formulons des revendications, ils nous traitent de subversifs. Ils nous disent que ce sont des sujets politiques, mais c'est évident. Nous avons droit à nous occuper de politique ou cela serait réservé au Gouvernement? »23. (Pulso, 2000)

En l'occurrence, I'argumentation étatique se justifie par l'ambition néolibérale de vouloir représenter la nature des choses, c'est-à-dire d'être vierge de toute référence idéologique. Par opposition à cette logique, les mouvements sociaux inscrivent leur action dans l'ordre politique.

\section{LA POLITISATION DES MOUVEMENTS SOCIAUX}

René-Antonio Mayorga parle pour qualifier la nouvelle conjoncture issue des élections de 2002 de «la fuerte politización de conflictos económicos y sociales» (Mayorga, 2002). De fait, la critique du néolibéralisme comme modèle de développement économique donne une

20 Alfonso Hinojosa, professeur de l'Université de Tarija, entretien réalisé par Marianne Raous dans le cadre d'un atelier de formation NINA, Tarija, juillet 2004 (Raous, 2004).

21 Pendant les bloqueos de septembre 2000, Banzer déclare «La concertación no será afectada por los intolerantes que han buscado el camino de la violencia para solucionar los problemas de la patria. ». Le président qualifie la demande d'Evo Morales d'arrêter l'éradication de la coca d'immorale. «Eso es inegociable e irreversible. Quienes están en contra de la erradicación están con el narcotráfico. » (La Prensa, 29 septembre 2000)

22 Entretien avec Oscar Olivera réalisé par Erick Fajardo Pozo en novembre 2003 (Fajardo, 2003).

23 Paysan de Ajllata Grande sur I'altiplano de Bolivie. 
dimension politique aux revendications. Elle est encore plus clairement assumée à partir de la proposition concernant la convocation d'une Assemblée Constituante. Celle-ci serait en effet en mesure de remettre en question l'ensemble du système politique en vigueur. Sur une affiche de la Coordination, on pouvait lire:

«Asamblea constituyente / para la participación de la gente / sin mediación partidaria / para decidir el país / en el que queremos vivir. » (Alenda, 2004).

Le terme de « politisation » est complexe et son acception extrêmement variable dans les théories de sciences politiques. Retenons la définition de Jacques Lagroye selon laquelle la politisation est « une requalification des activités sociales les plus diverses, requalification qui résulte d'un accord pratique entre des agents enclins, pour de multiples raisons, à transgresser ou à remettre en cause la différenciation des espaces d'activités. » (Lagroye, 2003 : 361-362). Le concept de Lagroye nous paraît particulièrement idoine pour éclairer notre propos dans la mesure où nous cherchons à comprendre le passage entre mouvement social — dont les membres pris collectivement sont par ailleurs assignés par l'État à un espace d'activité dépolitisé - et parti politique qui implique un autre espace jusqu'à présent très exclusif. Lagroye prend d'ailleurs l'exemple de la distinction des activités politiques et des activités syndicales à la fin du XIX' siècle en France, pour illustrer la segmentation des pratiques sociales.

Aux yeux de l'institution libérale, confondre les deux arènes relève bien d'une transgression puisqu'elle postule une différence essentielle entre l'action politique et les méthodes «fondamentalement extra-institutionnelles et particularistes du mouvement social. » (Lagroye, 2003 : 362). L'activité parlementaire ne saurait être soumise à une conception syndicale. Cette « architecture » induit donc la distinction de deux types de représentation : d'une part, l'organisation sociale qui revendique la représentation de secteurs mobilisés ; d'autre part, le parti politique qui doit être en mesure d'assurer une articulation entre les différents secteurs sociaux et ainsi produire une vision nationale. Les paradoxes de la participation que nous avons exposés plus haut illustrent la concomitance entre l'assignation à la sphère corporative, et la dénonciation par l'État de sa transgression. En tant que représentant de mouvements sociaux compris comme sectoriels, le reproche fait aux partis antisystèmes, en dehors de leur radicalité, est de ne représenter que leur propre secteur : les cocaleros pour Evo Morales, les Aymaras de I'altiplano pour Felipe Quispe.

Quelles sont donc les «raisons» qui poussent les acteurs à «transgresser ou à remettre en cause » les différenciations officiellement imposées. Plusieurs facteurs hétérogènes déterminent la politisation parmi lesquels se distinguent les facteurs historique, social, identitaire.

Commençons par analyser le facteur historique bien identifié. L'oscillation entre le formalisme parlementaire et la pression extraparlementaire est une des caractéristiques de la politique bolivienne. La révolution de 1952 et l'expérience du co-gobierno MNR/COB constitue un précédent quant à l'articulation entre le syndicat et l'État. De fait, le syndicat était alors paraétatique et constituait une des instances sur laquelle s'appuyait le parti hégémonique. La COB s'est toujours assumée comme une instance de représentation d'intérêts politiques24. L'émergence du MAS et du MIP serait-elle le signe de la continuation d'une indétermination historique entre les deux « ordres d'activité »?

Par ailleurs, dans sa recherche des raisons de l'instabilité politique en Bolivie avant 1982, JeanPierre Lavaud identifie «la politisation [comme résultant] de l'industrie des places publiques. Elle ne se limite pas aux catégories dominantes, [mais] regarde l'ensemble de la société ». Par-là I'auteur qualifie la Bolivie, "à l'instar de Samuel Huntington, [...] de société prétorienne de masse, pour signifier que non seulement l'armée est politisée, mais aussi les universités, les syndicats, le clergé... » (Lavaud, 1991 : 277).

24 La COB avait des «ministres ouvriers», et des représentants au sein de la direction de l'entreprise étatique Corporación Minera de Bolivia (Comibol) pouvant exercer leur droit de veto sur la politique minière du gouvernement. 
En ce qui concerne le facteur social de la structure communautaire, on peut d'ores et déjà relever que dans bien des cas, la mobilisation est facilitée par la préexistence d'une telle structure comme en témoigne l'organisation de bloqueo. Par ailleurs, on constate qu'en milieu urbain, cette logique n'a pas nécessairement disparue. Pour Sian Lazar (2004), la démocratie pratiquée à El Alto ne correspond pas à sa version libérale puisqu'il existe un plus ou moins grand degré d'obligation à la participation. L'organisation par quartiers25, à travers la Federación de Juntas Vecinales de El Alto (FEJUVE) a montré son efficacité pendant les journées sanglantes d'octobre pour coordonner les rondes de surveillance et l'assistance à la population.

Les individus ont l'habitude de se retrouver et de prendre des décisions ensemble. La participation à la vie collective n'est pas nécessairement perçue comme relevant d'un engagement politique qui serait séparé du reste de la vie sociale. La distinction de Tönnies entre Gemeinschaft (communauté) et Gesellschaft (société) (Tönnies, 1977) peut nous permettre de mieux comprendre cette proximité entre le social et le politique en Bolivie. La prégnance de la Gemeinschaft explique en effet que la charge assumée au nom de la collectivité ne soit pas politique au sens où on l'entend dans une société moderne, mais comprise comme partie intégrante de la vie civile. La mobilisation collective lors d'un bloqueo est en grande partie conditionnée par des déterminations communautaires. On retrouve cette dimension dans le syndicat. Derrière sa structure apparemment moderne, affichée depuis la révolution de 1952, sa base continue bien souvent de fonctionner selon une organisation de type communautaire, où les charges sont obligatoires, rotatives, et non électives (Albo, 2002). Ceci est surtout valable dans le cas de l'altiplano où l'on observe une « certaine symbiose entre les charges traditionnelles et syndicales. » (Albo et al., 1995 : 53), et moins dans celui des syndicats cocaleros, en partie issus des syndicats miniers.

Envisageons à présent le facteur identitaire. Selon Michel Dobry, il convient de s'intéresser à l'état conjoncturel des structures, aux propriétés contextuelles des configurations sociales, et à la manière dont celles-ci jouent sur la structuration des identités (Dobry, 1992). II rappelle l'aspect multidimensionnel de l'identité personnelle, et le fait que durant les périodes de transitions démocratiques, périodes de crises, les mobilisations collectives sont caractérisées par une tendance à la simplification de cette identité, à une régression vers l'habitus souvent utilisée par les entrepreneurs identitaires dans leurs stratégies de mobilisations antagonistes.

La revendication identitaire constitue une dimension essentielle des mouvements sociaux boliviens. Les mobilisations se déroulent à un moment où faire valoir ses origines indigènes constitue un plus, assure une reconnaissance auprès de la communauté internationale mais aussi de l'opinion publique et contribue par-là à la légitimation du mouvement. Alors même que l'assimilation par le métissage était la voie qui prévalait depuis 1952 dans la construction de la Nation, aujourd'hui les Indiens en tant que tels réclament leur intégration au corps politique. L'Indien réinventé devient le nouveau sujet de la revendication. La rhétorique de la participation et de la prise en compte de la diversité culturelle fait de l'identité un «recurso estratégico oportuno para fines tan diferenciados como lograr un cargo político, defender la hoja de coca, afirmar al grupo o negar al otro. » (Albó, 2002 : 100) .

Le passage d'un «espace d'activité » à un autre représente donc une transgression en ce qu'il consiste en un acte de politisation refusé à ceux qui l'accomplissent. Or, la définition que Frédéric Sawicki donne du « milieu partisan » identifie ce dernier à la résultante de circulations entre des espaces plus perméables les uns aux autres. II le définit comme "I'ensemble des relations consolidées entre des groupes dont les membres n'ont pas forcément comme finalité principale de participer à la construction du parti quoiqu'ils y contribuent en fait par leurs activités. » (Sawicki, 1997 : 24). Cette assertion permet ainsi de valider le concept de Lagroye relativement à la constitution d'un champ politique. On pourrait donc émettre I'hypothèse d'après laquelle les mobilisations de milliers de personnes œuvrent, comme par ricochet, en faveur de la constitution d'un «milieu partisan. ».

\footnotetext{
25 Ceux-ci portent souvent le nom de la région d'origine de la majorité des habitants qui s’y sont installés.
} 
Au total, les mouvements sociaux s'appuient sur un mode d'articulation pourtant disqualifié entre la société et l'État, qui joue un rôle essentiel non seulement dans leur propre formation mais également dans celle des partis qui nous intéressent. Les leaders respectifs du MAS et du MIP ne sont-ils pas tous les deux issus des rangs de la CSUTCB ?

Par ailleurs, à la montée en puissance des mouvements sociaux, répond une militarisation parfois drastique des territoires. Le Chapare en est le meilleur exemple. De ce point de vue « La historia de enemistad entre el ejército y las masas populares » semble se rejouer.

«La consolidación de los movimientos sociales, de sus propuestas políticas y su impacto en las decisiones es indesligable de la contundencia y densidad de medidas de presión como los bloqueos, las largas marchas y la confrontación con los cuerpos policiales y militares. 》 (Stefanoni, $2004: 22$ )

La rhétorique conciliatrice de la démocratie pactée est donc restée sans effet car ce nouveau scénario de confrontation s'apparente fortement à celui de 1952 même s'il faut observer plusieurs réserves. En particulier, se constituer en partis pour le MIP et pour le MAS pourrait représenter un gage de la reconnaissance du compromis démocratique. Ce n'est sans doute pas vrai de tout parti antisystème. Pensons à la stratégie légaliste du NSDAP26 de 1925 à 1933. Il faut donc finement distinguer dans les stratégies de chacun des deux partis les intentions et les actes qui offrent l'assurance d'une volonté démocratique authentique. Entre le MAS et le MIP, lequel des deux adopte une stratégie visant véritablement à « jouer avec » et non pas tout à fait « contre» les institutions?

Le vocable de « parti », dans la logique antisystémique, pourrait se comprendre comme le résultat d'un jeu rhétorique. II n'en provoque pas moins des effets réels. Ici l'expression «dire, c'est faire » se vérifie ${ }^{27}$. Le statut de parti politique semble toujours pouvoir être remis en question et abandonné au profit d'un retour aux origines : les organisations sociales (syndicat, coordination, etc). Le départ du parlement de Felipe Quispe en juin 2004, et ses déclarations sur l'erreur que constituerait la formation d'un parti en est une illustration. Cette indétermination qui confine à l'ambivalence semble ainsi constituer le caractère principal des partis «antisystèmes ». Au contraire, l'évolution du MAS en direction des institutions lui fait perdre cette dimension et le rapproche de plus en plus d'un parti traditionnel. C'est comme s'il existait deux bornes, et un continuum entre l'organisation de base et le parti politique formalisé. Dans ce cadre, c'est la relation avec l'État qui détermine pour une part essentielle la position politique du parti. Le rapprochement du MAS avec le gouvernement issu de la crise d'octobre illustre cette volonté de se positionner en tant que parti de gouvernement. Evo Morales reconnaît s'appuyer sur une légitimité double. Au lendemain des élections de 2002, il déclare :

«Somos la primera fuerza política del país, más que el MNR que probablemente tome el gobierno, porque contamos con dos factores decisivos: la legalidad que nos dieron las ánforas y la legitimidad social que nos dieron las movilizaciones de los sindicatos, las federaciones campesinas, los gremiales, las centrales obreras departamentales, entre tantas otras organizaciones. » (Chávez, 2002)

En conclusion, on peut noter avec Michel Offerlé que les partis sont « les résultats de multiples bricolages aléatoires par lesquels des entrepreneurs politiques se trouvant là pour des raisons sans doute très diverses, se produisent comme hommes politiques, tout en produisant les groupes qu'ils réunissent. »(Offerlé, 2002 : 40). Ainsi, pour Javier Albó, «l'élément le plus

\footnotetext{
26 Nationalsozialistische Deutsche Arbeiterpartei.

27 En 1952 les Ayllus se sont intégrés à des syndicats pour pouvoir accéder aux bénéfices de la Réforme agraire. Dans notre cas on pourrait dire que le glissement sémantique remplace le «syndicat » pour le « parti ». Réciproquement, l'évolution institutionnelle peut expliquer qu'une organisation indigène comme la CONAMAQ ait préféré prendre le nom de Consejo Nacional plutôt que celui de syndicat.
} 
Les partis « antisystèmes » en Bolivie : une remise en cause de l'architecture des rapports sociaux

novateur de ces dernières années a été la façon dont, à partir des organisations rurales, le mouvement s'est étendu jusqu'à inclure d'autres secteurs populaires. » (Albo, 2004 : 55). Le MIP et le MAS sont parvenus à cristalliser et formaliser la contestation. Se revendiquant d'un « contrôle social » exercé par les bases sur les dirigeants, ils construisent une image opposée à celle des élites politiques déconnectées des réalités de la «Bolivie d'en bas ». Ils en tirent une légitimité alternative fondée sur une représentation descriptive : «los semejantes votan por los semejantes ». Ce slogan renvoie-t-il à l'ordre identitaire, social, corporatif ou à la combinaison des trois ? Dans le cas du MIP, I'Indien constitue la figure exclusive de l'identité politique. Le MAS, quant à lui, ne cherche-il pas à gérer la fiction de la représentation sur un mode renouvelé ? Son discours permet en tous cas de fédérer des identités aussi bien indigènes que métisses, rurales qu'urbaines, pauvres qu'issues des classes moyennes et des intellectuels. Il s'appuie notamment sur une réalité bolivienne dans laquelle la négociation semble toujours possible.

\section{Références citées}

ALBÓ, X., 2002 - Pueblos indios en la política ; La Paz: Plural-CIPCA.

ALBÓ, X., 2004 - 222 años después, la convulsionada Bolivia multiétnica. Artículo primero ; Santa Cruz: CEJIS. Numéro spécial édité par V. Sandoval \& G. Herta.

ALBÓ, X., TICONA, E. \& ROJAS, G., 1995 - Votos y Wiphalas, campesinos originarios en democracia, 239 p. ; La Paz : CIPCA, Cuarderno de investigación 43.

ALENDA, S., 2004 - Los límites de la consolidación institucional. D’Orbigny, n 0, Mars: 26-40 ; La Paz.

ASSIES W., 2001 - David fights Goliath in Cochabamba: Water rights, neoliberalism and the renovation of social protest in Bolivia. T'inkazos, $\mathbf{n}^{\circ} \mathbf{8}$, Février: 179-205.

BARNADAS, J., 1983 - Actos de fe, Autos de fe, 384 p. ; Cochabamba: s.e.

CALLA, R., 1993 - Hallu hayllisa huti. Identificación étnica y procesos políticos en Bolivia (19731991). In : Democracia, etnicidad y violencia política en los países andinos (A. Adrianzén et al., eds.) : 57-81 ; Lima : Instituto de Estudios Peruanos-IFEA.

CALLA, R., 2000 - Los sindicalismos bolivianos contemporáneos: crisis y secundarización de un movimiento social desarticulado. In : Los moviemientos sociales en las democracias andinas (J. Massal \& M. Bonilla eds.) : 215-227 ; Quito ; Flasco Ecuador, IFEA.

CALDERÓN GUTIERREZ, F., 1999 - Actores sociales. In : Bolivia en el Siglo XX. La Formacion de la Bolivia Contemporanea. Analisis de la Reciente Historia de Bolivia (F. Campero Prudencio ed.) : 427-450 ; La Paz : Harvard Club de Bolivia.

CALDERÓN, F. \& LASERNA, R., 1989 - Nación, Estado y movimientos sociales regionales en Bolivia (1917-1983). In : Movimientos populares en América Latina (D. Camacho \& R. Menjivar, éds.) : 294-307 ; México : Siglo XXI.

CHÁVEZ, W., 2002 - Dos Bolivias, dos poderes. Juguete Rabioso, 59 : 8-9 ; La Paz.

CHAZEL, F. (éd.), 1993 - Action collective et mouvements sociaux, 267 p. ; Paris : PUF.

COUFFIGNAL, G., 2003 - Les démocraties latino-américaines sont-elles gouvernables ? In : Être gouverné, études en l'honneur de Jean Lecas (P. Favre, J. Hayward \& Y. Schemeil, éds.) : 225-241; Paris : Presses de la Fondation Nationale des Sciences Politiques.

CRABTREE, J. \& WHITEHEAD, L., 2000 - Towards democratic viability: the Bolivian experience, 240 p. ; New York : Palgrave.

DOBRY, M., 1992 - Sociologie des crises politiques, 319 p. ; Paris : Presses de la Fondation Nationale des Sciences Politiques.

GOLSTONE, J., 2003 - Introduction: Bridging Institutionalized and Noninstitutionalized Politics. In : State, Parties and Social Movements : 1-26; Cambridge: Cambridge University Press. 
GUZMAN, G. \& ORDUNA, V., 2002 - Evo Morales y Felipe Quispe, los votos inesperados. Pulso, $\mathbf{n}^{\circ} 149$ : 4.7 juin.

LAGOS, M. L, 2001 - Bolivia la nueva: la construcción de una nueva ciudadanía. In : Visiones de fin de siglo. Bolivia y América latina en el siglo XX (D. Cajías, M. Cajías, C. Johnson \& I. Villegas, éds) : 271-280 ; La Paz : Iféa-Coord. de Historia-Embajada de España en Bolivia.

LAGROYE, J., 2003 - Les processus de politisation. In : La Politisation : 359-372 ; Paris : Belin.

LA PRENSA, 2000 - « Eso es inegociable e irreversible. Quienes están en contra de la erradicación están con el narcotráfico », 29 septembre ; La Paz.

LA PRENSA, 2002 - 11 janvier; La Paz.

LA RAZÓN, 2003 - «El Presidente quiere evitar un concurso de acreedores », $1^{\text {er }}$ janvier ; La Paz.

LAVAUD, J.-P., 1991 - L'instabilité politique de l'Amérique Latine, le cas de la Bolivie, 290 p. ; Paris : IHEAL-L'Harmattan.

LAVAUD, J.-P., 1993 - Conflictos sociales y democracia en Bolivia . In : Democracia, etnicidad y violencia política en los países andinos (A. Adrianzén et al., eds.) : 43-53 ; Lima : Instituto de Estudios Peruanos-IFEA.

LAZAR, S., 2004 - Mobilización social en El Alto. Communication présentée au Colloque «Democracia, gobernabilidad y participación en Bolivia, 1993-2003 » : 25-26 mars ; Samanca : Instituto de Iberoamérica y Portugal.

LINZ, J., 1990 - Transitions to Democracy. Washington Quaterly, 13, nº 3 : 143-164.

MAYORGA, R. A., 2002 - «Las elecciones de 2002 y la metamórfosis del sistema de partidos en Bolivia ». http://www.observatorioelectoral.org

NEVEU, E., 2002 - Sociologie des mouvements sociaux, 125 p. ; Paris : La découverte.

O'DONNELL, G., 1996 - Poverty and inequality in Latin America: some political reflections. Working Paper, 225 : 17 p. ; University of Notre Dame : Helen Kellogg Institute for International Studiese, juillet.

OFFERLÉ, M., 2002 - Les partis politiques, 128 p. ; Paris : PUF, 4e édition.

OLIVERA, O., 2003 - « De Febreo negro al Octubre rojo. Crónica de un año de agonía neoliberal en las calles de Bolivia ». Entretien réalisé par Erik Fajardo. http://www.rebelion.org

PULSO, 2000 - El bloqueo sigue en Archacachi. Pulso, $\mathbf{n}^{\circ}$ 38: 18. 14 avril.

RAOUS, M., 2004 - Agir entre préséances et préférences normatives : la mobilisation indigène en Bolivie. Mémoire de DEA, Paris I.

SAWICKI, F., 1997 - Les réseaux du Parti socialiste. Sociologie d'un milieu partisan, 335 p. ; Paris : Belin.

SORIA SORAVIA, M., 2002 - Democracia e izquierda en Bolivia, 218 p. ; La Paz : Ed. Artes gráficas Sagitario.

STEFANONI, P., 2004 - Articulando lo político y lo social : Algunas reflexiones sobre el MASIPSP. Temas Sociales, Revista de Sociologia, $\mathbf{n}^{\circ} 25$ : 15-45 ; La Paz : UMSA,. p..

TARROW, S., 1997 - Power in movement: social movements, collective action and politics, 270 p. ; Cambridge : Cambridge university Press.

TÖNNIES, F., 1977 - Communauté et société : catégories fondamentales de la sociologie pure, 285 p. ; Paris : Retz. 